\title{
Fractal Statistics
}

\author{
B.G. Sidharth* \\ Centre for Applicable Mathematics \& Computer Sciences \\ B.M. Birla Science Centre, Adarsh Nagar, Hyderabad - 500063 (India)
}

\begin{abstract}
We consider the recent description of elementary particles in terms of Quantum Mechanical Kerr-Newman Black Holes, a description which provides a rationale for and at the same time reconciles the Bohmhydrodynamical formulation on the one hand and the Nelsonian stochastiic formulation on the other. The Boson-Fermion divide is discussed, and it is pointed out that in special situations, anomalous statistics, rather than Bose-Einstein or Fermi-Dirac states, can be encountered.
\end{abstract}

\section{Introduction}

In a recent model[迎, 2] Fermions, in particular the electrons have been treated as, what may be called Quantum Mechanical Kerr-Newman Black Holes (QMKMBH), a treatment that leads to a successful interpretation of several hitherto inexplicable features, both in Particle Physics and Cosmology [3, 4, 5, 6]. In the hydrodynamical formulation these QMKNBH can be considered to be vortices bounded by the Compton wavelength in a description that leads to a harmonious convergence with Nelson's stochastic theory also [7] (Cf.Discussion). In this picture, Bosons would be, not vortices, but rather streamlines [8].

In what follows, we shall show that within this framework it is possible to explain the divide between Fermi-Dirac and Bose-Einstein statistics, as also examine special situations where there would be fractal statistics, that is Bosonisation of Fermions and Fermionisation of Bosons.

\footnotetext{
0*Email:birlasc@hd1.vsnl.net.in; birlard@ap.nic.in
} 


\section{Usual Statistics}

We first observe that the above vortex and streamline description provides an explanation for the Fermionic and Bosonic statistics. Indeed, let $n_{K}$ be the occupation number for the energy or momentum state defined by $K$. For Fermions $n_{K}=0$ or 1 , where as $n_{K}$ can be arbitrary for Bosons. The reason is that Fermions are bounded by the Compton wavelength. That is, they are localised, a description which requires both negative and positive energy solutions [9], which infact is expressed by zitterbewegung effects. The localisation in space automatically implies an indeterminacy of energy or momentum $K$. Thus, though an energy or momentum state, in practical terms implies a small spread $\Delta K$, it is not possible to cram Fermions which also have a momentum energy interdeterminacy spread, arbitrarily into this state.

On the other hand Bosons are not bound by the Compton wavelength vortices, and so have sharper momentum states, so that any number of them can be crammed into the state $K$ (which really is blurred by the Uncertainity Principle indeterminacy of $\Delta K)$. Another way of expressing these facts are by saying that the Fermionic wave function in space is weak, but not the Bosonic wave function [10], the latter fact being symptomatic of a field or an interaction.

The above considerations immediately follow from a recent description in terms of quantized fractal space time 11]. In this case we have a non commutative geometry given by

$$
[x, y]=0\left(l^{2}\right),\left[x, p_{x}\right]=\imath \hbar\left[1+l^{2}\right]
$$

where $l$ is the Compton wavelength. It is precisely the space quantization at the Compton scale that leads to the Dirac matrices and their anticommutation relations.

Bosons on the other hand would be bound states of the Fermions (Cf. 112 and

other references), or alternatively they would be a super position of vortices leading to a streamline like description [8]. 


\section{Fractal Statistics}

However there could be certain special situations in which the above space localised and momentum space localised description of Fermions and Bosons gets blurred, in which case anomalous or fractal statistics would come into play. This could happen, for example when the Compton wavelength $l$ of the Fermion becomes very small, that is the particle is very massive. In this case the non commutativity of the geometry referred to above in (1) disappears and we return to the usual commutation relations of non relativistic Quantum Mechanics, that is a description in terms of the spinless Schrodinger equation. Indeed in this case, $v$ being the velocity of the particle $v / c$ would be small and the Dirac equation tends to the Schrodinger equation[9].

There would thus be a Bosonisation effect. This would also be expected at very low temperatures, for example below the Fermi temperature, when the energy spread of the Fermions would itself be small, rather as in the case of Bosons and anomalous behaviour, for example on the lines of the superfluidity of $H e^{3}$ 13 can be expected.

For very light Fermions, for example Neutrinos, the Compton wavelength be very large, but in this case the double connectivity of the QMKNBH disappears and the observed anomalous features of the Neutrino show up, as discussed elsewhere [1, 14].

Indeed with recent developments in nano technology and thin films, we are able to consider one dimensional and two dimensional Fermions, in which case, Bosonisation effects show up as discussed elsewhere[13]. In any case in the one dimensional and two dimensional cases, the Dirac equation becomes a two component equation, without an invariant mass 15, while at the same time, handedness shows up [16]. It is worth mentioning here that the Dirac matrices can have only even dimensionality, corresponding to the above anomalous two component Dirac spinors, and the usual Dirac bispinors of the three dimensional theory.

We now consider in greater detail two illustrative situations where anomalous behaviour shows up.

1. Nearly Mono Energetic Fermions (Cf.[[17]):

Our starting point is the well known formula for the occupation number of a Fermion gas 18

$$
\bar{n}_{p}=\frac{1}{z^{-1} e^{b E_{p}}+1}
$$


where, $z^{\prime} \equiv \frac{\lambda^{3}}{v} \equiv \mu z \approx z$ because, here, $\mu \approx 1$ (Cf. Appendix);

$$
\begin{gathered}
v=\frac{V}{N}, \lambda=\sqrt{\frac{2 \pi \hbar^{2}}{m / b}} \\
b \equiv\left(\frac{1}{K T}\right), \quad \text { and } \sum \bar{n}_{p}=N
\end{gathered}
$$

Let us consider particular a collection of Fermions which is somehow made nearly mono-energetic, that is, given by the distribution,

$$
n_{p}^{\prime}=\delta\left(p-p_{0}\right) \bar{n}_{p}
$$

where $\bar{n}_{p}$ is given by (2).

This is not possible in general - here we consider a special hypothetical situation of a collection of mono-energetic particles in equilibrium which is the idealization of a contrived experimental set up. For example, the following is a mono energetic equilibrium distribution function:

$$
f \alpha \exp \left[-\rho\left(|\vec{v}|-\left|\vec{v}_{0}\right|\right)^{2}\right], \rho \equiv m / 2 K T>>1,
$$

Infact one can show that the above function is consistent with the Bolitzmann equation for a collection of particles under a uniform magnetic field $\vec{B}$ : Infact

$$
\frac{\partial f}{\partial t}=0
$$

while one can easily show that

$$
\vec{\Delta} f=0, \vec{\Delta}_{\vec{v}}\left[\exp \left[-\rho\left(|\vec{V}|-\left|\vec{V}_{0}\right|\right)^{2}\right] \alpha\left[\left(|\vec{V}|-\left|\vec{V}_{0}\right|\right) f \vec{V}\right]\right.
$$

Further the force is given by

$$
\vec{F} \alpha \vec{V} \times \vec{B}
$$

so that the force term in the Boltzmann equation viz.,

$$
\frac{\vec{F}}{m} \cdot \vec{\Delta}_{\vec{v}} f \alpha \vec{V} \times \vec{B} \cdot \vec{V}=0
$$

Finally

$\frac{\partial f}{\partial t}=0$ means $\frac{d H}{d t}=0$ that is $\left(\frac{\partial f}{\partial t}\right)_{\text {coll }}=0$ 
Thus the above distribution function which becomes mono energetic for $\lambda>>$ 1 satisfies the Boltzmann equation (Cf.ref. 18); when $\rho \rightarrow \infty$ we get the above $n_{p}^{\prime}$ in this case.

By the usual formulation we have,

$$
N=\frac{V}{\hbar^{3}} \int d \vec{p} n_{p}^{\prime}=\frac{V}{\hbar^{3}} \int \delta\left(p-p_{0}\right) 4 \pi p^{2} \bar{n}_{p} d p=\frac{4 \pi V}{\hbar^{3}} p_{0}^{2} \frac{1}{z^{-1} e^{\theta}+1}
$$

where $\theta \equiv b E_{p_{0}}$.

It must be noted that in (5) there is a loss of dimension in momentum space, due to the $\delta$ function in (田) - infact such a fractal two dimensional situation would in the relativistic case lead us back to the anomalous behaviour already alluded to 19. In the non relativistic case two dimensions would imply that the coordinate $\psi$ of the spherical polar coordinates $(r, \psi, \phi)$ would become constant, $\pi / 2$ infact. In this case the usual Quantum numbers $l$ and $m$ of the spherical harmonics 20] no longer play a role in the usual radial wave equation

$$
\frac{d^{2} u}{d r^{2}}+\left\{\frac{2 m}{\hbar^{2}}[E-V(r)]-\frac{l(l+1)}{r^{2}}\right\} u=0,
$$

The coefficient of the centrifugal term $l(l+1)$ in (6) is replaced by $m^{2}$ as in Classical Theory 21].

To proceed, in this case, $K T=<E_{p}>\approx E_{p}$, so that, $\theta \approx 1$. But we can continue without giving $\theta$ any specific value.

Using the expressions for $v$ and $z$ given in (3) in (4), we get

$$
\begin{aligned}
& \left(z^{-1} e^{\theta}+1\right)=(4 \pi)^{5 / 2} \frac{z^{\prime}-1}{p_{0}} ; \text { whence } \\
& z^{\prime-1} A \equiv z^{\prime-1}\left(\frac{(4 \pi)^{5 / 2}}{p_{0}}-e^{\theta}\right)=1
\end{aligned}
$$

where we use the fact that in (3), $\mu \approx 1$ (Cf.Appendix). A number of conclusions can be drawn from (7). For example, if,

$$
\begin{gathered}
A \approx 1, i . e ., \\
p_{0} \approx \frac{(4 \pi)^{5 / 2}}{1+e}
\end{gathered}
$$


where $A$ is given in (7), then $z^{\prime} \approx 1$. Remembering that in (3), $\lambda$ is of the order of the de Broglie wave length and $v$ is the average volume occupied per particle, this means that the gas gets very densely packed for momenta given by (8). Infact for a Bose gas, as is well known, this is the condition for Bose-Einstein condensation at the level $p=0$ (cf.ref. [18]).

On the other hand, if,

$$
\left.A \approx 0 \text { that is } \frac{(4 \pi)^{5 / 2}}{e} \approx p_{0}\right)
$$

then $z^{\prime} \approx 0$. That is, the gas becomes dilute, or $V$ increases.

More generally, Equation (7) also puts a restriction on the energy (or momentum), because $z^{\prime}>0$, viz.,

$$
\begin{gathered}
A>0\left(\text { i.e. } p_{0}<\frac{(4 \pi)^{5 / 2}}{e}\right) \\
\text { But } \quad \text { if } A<0,\left(\text { i.e. } p_{0}>\frac{(4 \pi)^{5 / 2}}{e}\right)
\end{gathered}
$$

then there is an apparent contradiction.

The contradiction disappears if we realize that $A \approx 0$, or

$$
p_{0}=\frac{(4 \pi)^{5 / 2}}{e}
$$

(corresponding to a temperature given by $K T=\frac{p_{0}^{2}}{2 m}$ ) is a threshold momentum (phase transition). For momenta greater than the threshold given by (9), the collection of Fermions behaves like Bosons. In this case, the occupation number is given by

$$
\bar{n}_{p}=\frac{1}{z^{-1} e^{b E_{p}}-1},
$$

instead of (2), and the right side equation of (7) would be given by ' -1 ' instead of +1 , so that there would be no contradiction. Thus in this case there is an anomalous behaviour of the Fermions.

The Bosonic behaviour of Fermions can be understood in a simple way from a different standpoint. Let us consider the case described before equation (5), of a collection of Fermions with $m>>1$, and consequently all having nearly the same momentum $\vec{p}$. In this case, it is possible to choose a Lorentz 
frame in which all the particles are nearly at rest, i.e., $v / c \approx 0$. As mentioned, it is known that the Dirac equation describing spin $1 / 2$ particles goes over into the Schrodinger equation describing spinless particles[9]. Effectively, we have a collection of Bosons.

\section{Degenerate Bosons:}

We could consider a similar situation for Bosons also (Cf. 22] where an equation like (4) holds. In this case we have equations like (8) and (9):

$$
\begin{gathered}
p_{0} \approx \frac{(4 \pi)^{5 / 2}}{1.4 e-1} \\
p_{0} \approx \frac{(4 \pi)^{5 / 2}}{e}
\end{gathered}
$$

((11) is the same as (9), quite expectedly). At the momentum given by (10) we have a densely packed Boson gas rather as in the case of Bose Einstein condensation. On the other hand at the momentum given by (11) we have infinite dilution, while at lower momenta than in (11) there is an anomalous Fermionisation.

Finally it may be pointed out that at very high temperatures, once again the energy - momentum spread of a Bosonic gas becomes large, and Fermionisation can be expected, as in indeed has been shown elsewhere [23]. In any case at these very high temperatures, we approach the Classical Maxwell Boltzmann situation.

\section{Discussion}

1. It was mentioned in Section 1 that in the QMKNBH description there is a reconciliation between Nelson's stochastic theory and the Bohm-Hydrodynamical approach, once it is realized that the diffusion constant of Nelson's theory is related to the Compton wavelength, and that the non local Bohm potential gives the energy of the particle (Cf. [1], 7] for details). Infact in the Nelsonian theory, space time is a non differenciable manifold, there being a Double Weiner process which leads to the usual solinoidal velocity given by $\vec{v}=\frac{\vec{\nabla} S}{m}$ as also an osmotic velocity given by $\vec{u}=\frac{\hbar}{2 m} \frac{\vec{\nabla} R}{R}$ where the Quantum Mechanical wave function $\psi=R e^{\frac{2}{\hbar}} S$. We get identical expressions in 
the Bohm-hydrodynamical approach also. Indeed if there were no double Weiner process, then in the Nelsonian theory $\vec{u}$ would vanish and so would $\nabla^{2} R$ and $Q$, that is the Compton wavelength vortex and the mass and the energy of the particle would both disappear.

2. Interestingly in the QMKNBH-Hydrodynamical vortex picture as in the usual Quantum Theory of addition of angular momentum, we can recover the fact that the sum or bound state of two such vortices or spin half particles would indeed give Bosons.

This can be seen as follows, from the theory of vortices 24. The velocity distribution is given by

$$
v=\Gamma / 2 \pi r
$$

In the case of the QMKNBH vortex we have to use in (12) $v=c$ and $r=$ $\hbar / 2 m c$, the Compton wavelength of the particle. So we have $\Gamma=\frac{h}{m}$ which is also the diffusion constant of Nelsonian Theory.

If we consider two parallel spinning vortices separated by a distance $d$, then the angular velocity is given by

$$
\omega=\frac{\Gamma}{\pi d^{2}}
$$

whence the spin of the system turns out to be $h$, that is in usual units the spin is (四), wth states \pm 1 .

There is also the case where the two above vortices are anti parallel. In this case there is no spin, but rather there is the linear velocity given by

$$
v=\Gamma / 2 \pi d
$$

This corresponds to the spin 1 case with state 0 .

Together, the two above cases give the $3,-1,0,+1$ states of spin 1 as in the Quantum Mechanical Theory.

It must be noteed that the distance $d$ between vortices could be much greater than the Compton wavelength scale, so that the wave function of the Boson in the above description would be extended in space in comparison to the Fermionic wave function, as pointed out in the text.

\section{References}

[1] Sidharth, B.G., 1997 Ind.J. of Pure and Applied Phys., 35, pp456-471. 
[2] Sidharth, B.G., 1998 Int.J.Mod.Phys.A., 13(15), pp2599-2612.

[3] Sidharth, B.G., 1999 Mod.Phys.Lett.A., Vol.14 No.5, pp387-389.

[4] Sidharth, B.G., Gravitation \& Cosmology, Vol.4, No.2, 1998.

[5] Sidharth, B.G., 1997 Mod.Phys.Lett.A., Vol.12 No.32, pp2469-2471.

[6] Sidharth, B.G., International Journal of Theoretical Physics, 37 (4), 1307-1312, 1998.

[7] Sidharth, B.G., "Space Time as a Random Heap", to appear in Chaos, Solitons \& Fractals.

[8] Sidharth, B.G., 1999 in Proceedings of Eighth Marcel Grossmann Meeting on General Relativity, T. Piran and Remo Ruffini (Eds.), World Scientific, Singapore,pp479-481.

[9] J.D. Bjorken and S.D. Drell, "Relativistic Quantum Mechanics", McGraw-Hill Inc., New York, 1964.

[10] Schiff, L.I., "Quantum Mechanics", McGraw-Hill Book Company, Singapore, 1968, p.490.

[11] Sidharth, B.G., 2000 Chaos, Solitons and Fractals, 11 (8), p1269-1278.

[12] Finkelstein, D., Saller, H., and Tang, Z., "Gravity Particles and Space Time", Eds. P. Pronin, G. Sardanashvily, World Scientific, Singapore, 1996.

[13] Sidharth, B.G., Journal of Statistical Physics, 95(3/4), May 1999.

[14] Sidharth, B.G., "From the Neutrino to the edge of the Universe", to appear in Chaos, Solitons \& Fractals.

[15] A. Zee, "Unity of Forces in the Universe", Vol.II, World Scientific, Singapore, 1982, and several papers reproduced and cited therein.

[16] Heine, V.,"Group Theory in Quantum Mechanics", Pergamon Press, Oxford, 1960, p.364. 
[17] Sidharth, B.G., "A Note on Degenerate Bosons", CAMCS Technical Report 95-04-07b.

[18] Huang, K., 1975 "Statistical Mechanics", Wiley Eastern, New Delhi,pp230-237.

[19] Sidharth, B.G., "Interstellar Hydrogen" to appear in Chaos, Solitons \& Fractals.

[20] Powell, J.L., \& Crasemann, B., "Quantum Mechanics", Narosa Publishing House, New Delhi, 1988.

[21] Goldstein, H., 1966 "Classical Mechanics", Addison-Wesley, Reading, Mass., p76.

[22] xxx.lanl.gov/quant-ph/abs 9506002.

[23] Sidharth, B.G., Bull.Astr.Soc.India, 25, 1997.

[24] Donnelly, R.J., "Quantized Vortices in Helium II", Cambridge University Press, Cambridge, 1991, pg.13. 